

Cada compringido conkene coms principio activo: Seroxat $20 \mathrm{mg}$; paroxetina (en forma de clorhidrato) $20 \mathrm{mg}$. FORMA EARMACEUTICA: Seroxat se presénta en comprimidos recabientos, biconvexos DATOS CLINICOS: INOICACIONES TERAPEUTICAS Tratamiento de la depresión. Tratamiento preventivo de las recaidas y recidivas de la depresión. El tatamiento prolongado con paroxetina mantiene st eficcria durante periodos de hasta 1 año POSOLOGIAY FORM OE ADUMNSTRACION. Adutios La dosis recomentada es de 20 midía. En algunos pacientes, en furción de la respuest dínica, puede ser necesario aumentar la dosis. Se debe realizar gradualmente con incrementos de 10 mg (generalmente cada semana) hasta un máximo de $50 \mathrm{mg}$. Se recomienda administrar la paroxetina una vez al dia, por la mañana (a primera hora), con algo de alimento. El comprimido se debe deglutir y no masticar. Como con todos los tamacos antideppesivos, la dosificacion se debe eevisar, y ajustarar si es necesanio, a las 2.3 semanas de iniciado el tratamiento, si se considera clinicamente apropiado. Se reconienda continuar el tratamiento antidepresivo duarante un periodo suficiente, que habitualmente es de varios meses. A igual que con cualquier psicofámaco, es recomendable suspender la terapia gradualmente debido a la posible aparición de sintomas tales como initabilidad, alieraciones del sueño, vériço, agitación, ansiedad, náuseas y sudoración. La administración malutina de paroxetíra no autera ni la calidad ni la duración del sueño. Más aún, los pacientes suelen experimentar una meioria del sueno, a medida que responden al tatamiento con paroxeitra. Ancianos. La dosis recomencada es de

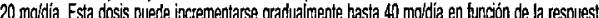
del paciente. Niños. Ver "Contraindicaciones". Pacientes con insufficiencia renal o henáfica: En los pacientes con insuticiencia renal (aclaramiento de creatinina <30minnin) o hepática grave, la dosis se debe reducis al límite inferior del rango terapestico $(20 \mathrm{mg})$. CONTRAINDICACIONES Hipersensibilidad conocida a paroxetina. No se recomienda el empleo de paroxetina en niños, ya que no se ha establecido la securidad y eficacia del lámaco en este grupo de población. ADVERTENCLAS PRECAUCIONES ESPECLALES DE EMPLEO: Entermedad cardiaca paroxetina no produce cambios dinicamente significativos de la presión arterial, trectencia cardiaca ni ECG. No obstante, es necesario tomar las precauciones habitules en los pacientes con cardiopatias. Enilensia como ocurre con otos antidepresiros, paroxetina debe emplearse con precaución en los pacientes epilépticos. Convulsiones. a experiencia incica que la ircidencia de convilsiones es menor del $0.1 \%$ en los pacientes tratados $c 0$ paroxelina. El tratamiento debe intenumpirse en todo pactente que desamolle crisis convulsivas. Terapin electroconvisiva, (TEC): existe muy poca experiencia dínica sobre la administración conconilante de paroxetina con TEC. Precauciones especiales Como ocurre con la mayoria de los antidepresivos, no se debe ưfilizar paroxetina en combinacion con los intibidores de la monoaminooxidasa (MAO). A igual que todos los antidepresivos, paroxetira se debe udilizar con precaución en pacientes con antecedenties de mania. Paroxetina debe administarse con precaución en los pacientes tratados con anticaagularites Orales. INTERACCION CON OTROS MEDICAMENTOS Y OTRAS FORMAS DE INTERACCION: La absorción y la farmacocinética de paroxetina no se modifican con alimentos ni antiácidos. La inducción e inhibición de los sistemas enzimábicos hepáticos, pueden atectar et melabolismo y la farmacocinética de paroxetina. Al igual que otros intibidores de la recaptación de 5.HT, los estuvios en animales indican que puede procuduise una interacción entre paroxetina y tipotólano dando como resullado el 'Sindrome de serotorina' que se traduce en una combinación de agiteción, insomrio y sintomas gastrointestinales que inciuven diarea. Aunque pasoxetina no aumenta el deterioro que provoca el alcohol sobre las funciones mental y molora, no se recomienda administrar simutáneamente paroxetina y alcohol en los pacientes deprimidos. La experiencia en un grupo de individuos sanos demuestra que paroxetina rio aumenta el efecto sedante ni la contusión asociada a halopenidol, amilobarital y oxazepam, cuando se adninista jurto con estos lámacos. La administración concomitante de paroxetina y antientépticos puede estar asociada a un mayor número de electos secundarios. EMBARAZO Y LACTANCA: No SE ha establecido la segunidad de paroxetina en mujeres embarazadas, por to que no debe udilizarse durante el embarazo o la lactancia, a menos que el posible benefico supere el nesgo potencia. EFECTOS SOBRE LA CAPACIDAD PARA CONDUCIR VEHICULOS Y UTILZAR MAOUINAS: LO experiencia círica demuestra que el tatamiento con paroxetina no produce atteracion de la función cognitva ni psicomotore. Sin embargo, como ocure con todos los psicotármacos, hay que advertir al paciente sobre la posibilidad de que se prodszcan alteraciones en la capacidad para conduci vehicalos y para manipular máquinas automáticas. EFECTOS INDESEABLES: Los electios indeseables de la paroxetina son en general de naturaleza leve y no modfican la calidad de vida del paciente. Estos etectos secundanios suelen disminuir en intensidad y frecuencia al continuar el tratamiento y en genera no obligan a suspender la medicación. Los electos secundarios más comunes asociados con el uso de paroxetina, que ro se chservaron con la misma incidencia en pacientes fratados can placebo, fueron a temblor, mistriasis, sequedad de boca e intabbidad. No se ha descrito ningún caso de anomalias del ECG, cona o convulsiones tras la sobredosis con paroxetina. Se han comunicado casos de sobredosis con paroxetina sola (hasta $850 \mathrm{mg}$ ) y en cambinación con otros agentes. Cuando se utilizo paroxetina sola, no se produjo ninguna muerte y la recuperación se llevó a cabo sin inconvenientes. El tratamiento comorende las medicas generales habituales utilizadzs trente a la sobredosis por cualavier antidepresino PAOPIEDADES FAPUACOLOGICIS: PPOPIEDADES FARUACODIMUICAS

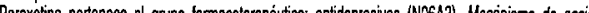
Paroxetina es un inhibicor potente y selectivo de la recaptación de 5 -hidroxitnotamina (5-HT serotonina). No es probable que los metabolitos de paroxetina contribuyan a la acción terapétitica dada la práctica ausencia de actividad farmacológica de los mismos. Electos fámacodinámicos. Paroxetina no produce alleración de la función psicomotora ni potencia el electo depresor del elanol. Los estudios en animales indican que paroxetina es bien tolerada a nivel del sistema cardiovascular, Paroxetina no produce en individuos sanos cambios clinicamente sionificativos de la presión arterial trecuencia cardiaca o ECG PROPIEDADES FARMACOCIMETICAS: Paroxetina se absorbe bien tras adninistración oral y surfe metabolismo de primer paso. La vida media de eliminación es variable, pero generalmente oscila alrededor de 24 horas. Los niveles sistémicos estabies se obtienen al cabo of 7.14 dias del iricio del tratamiento y la lamacocinética no se modifica durante tratamientos prolongados. A concentraciones terapérticas, aproximadamenta el $95 \%$ de la paroxetina presente en el plasma se une a las proteinas plasmáticas. No hay correlación entre las concentraciones plasmáticas de paroxetina y sU eficacia y tolerancia. DATOS PRECLINCOS DE SEGLRIDAD: Tras I administración oral en rata y ratón, la DL50 aguda de paroxetina es muy elevada (aprox. $350 \mathrm{mgkg}$ ) en relación con la dosis clinica. No se observó ningún efecto cancerigeno en la rata y el ratón; ni tampoco se apreciaron etectes genotoxicos en una serie de pruebas de midagenicidad in vitro e in vivo. Los esiudios de leratogenicidad realizados con conejos y ratas con dosis respectivas entre 6-15 y 52.130 veces slipeniores at rango terapéutico recomendado en clinica, no mostraron siznos de teraiogenicidad

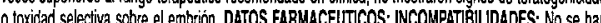

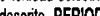
No requiere condiciones especiales. PRESENTACIONES Y PYP IVA 4\%: Envase tipo blister. Cada envase contene: Seroxat $20 \mathrm{mg}, 14$ comprimitos: 3.173 pts. Seroxat 20 mg, 28 comprimidos: 6.000 pts CONSULTE LA FICHA TECNCA COMPLETA DEL PRODUCTO ANTES DE PRESCRIBIR. 'Seroxal es marca registrada. Nombre y domicalio social del titular de la Autorización de Comercialización. SmithKline Beecham, S.A.C/ Valle de la Fuentria, 3-28034 Madrid. 


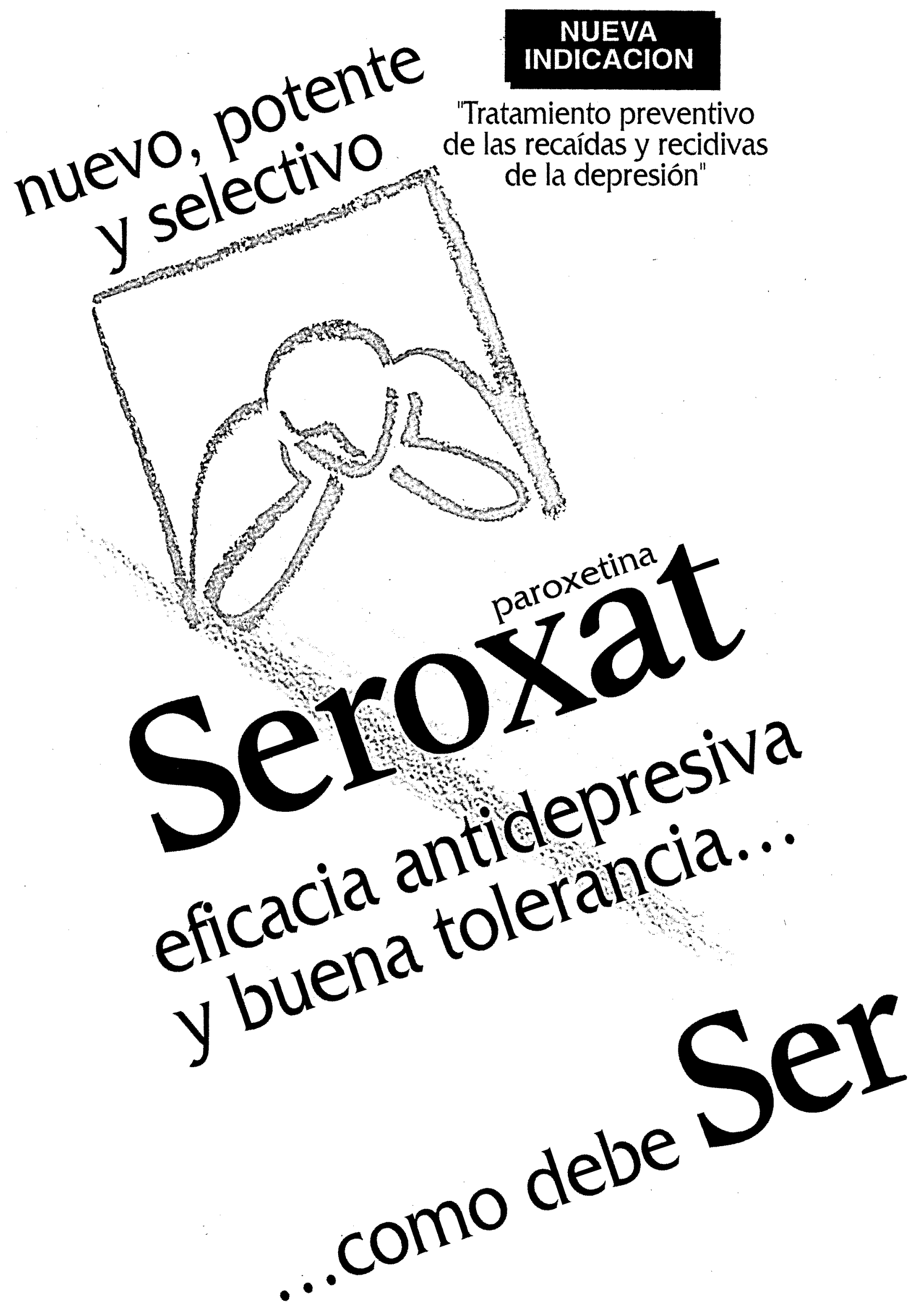




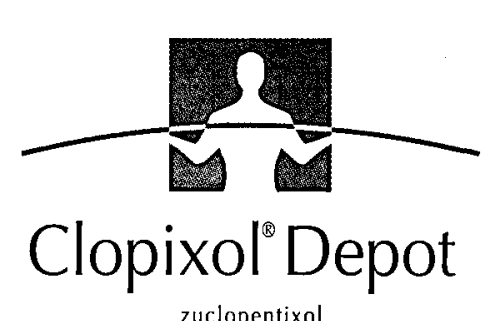

CLOPIXOL ${ }^{\circledR}$ Depot. Zuclopentixol (DCI). Clopixol ${ }^{\circledR}$ Depot, inyectable. COMPOSICION: Cada ampolia de $1 \mathrm{ml}$ contiene: zuclopentixol (DCI) decanoato, 200 mg. Excipiente: aceite vegetal. INDICACIONES: Tratamiento de la esquizofrenia crónica y subcrónica especialmente en pacientes en los que existe dificultad de establecer un cumplimiento por vía oral. POSOLOGIA: Adultos: La dosis y el intervalo de administración deberá ajustarse individualmente, para alcanzar una supresión máxima de los síntomas psicóticos con un mínimo de efectos indeseados. En el tratamiento de mantenimiento las dosis oscilarán entre 200 y $400 \mathrm{mg}$ cada 2 a 4 semanas. Algunos pacientes pueden necesitar dosis superiores o intervalos inferiores entre las dosis. La dosis máxima recomendada es de $600 \mathrm{mg}$ $(3 \mathrm{ml})$ por semana. Cuando se cambie el tratamiento de zuclopentixol aral o acetato de zuclopentixol intramuscular a tratamiento de mantenimiento con decanoato de zuclopentixol se seguirán las siguientes directrices: 1/ Cambio de zuclopentixol oral a decanoato de zuclopentixol: $25 \mathrm{mg} /$ día por vía oral equivalen a $200 \mathrm{mg}$ de decanoato de zuclopentixol cada 2 semanas; $25 \mathrm{mg} / \mathrm{dia}$ por via oral equivalen a $400 \mathrm{mg}$ de decanoato de zuclopentixol cada 4 semanas. 2) Cambio de acetato de zuclopentixol a decanoato de zuclopentixol: simultáneamente con la (últimal inyección de acetato de zuclopentixol $1100 \mathrm{mg}$ ), se administrarán $200-400 \mathrm{mg}(1-2 \mathrm{ml})$ de decanoato de zuclopentixol $200 \mathrm{mg} / \mathrm{ml}$. Las inyecciones de decanoato de zuclopentixol se repetirán cada dos semanas. Algunos pacientes pueden necesitar dosis superiores o intervalos inferiores entre las dosis. El acetato de zuclopentixol y el decanoato de zuclopentixol pueden mezclarse en una jeringa y administrarse como una sola inyección intramuscula (coinyección). Los pacientes que hayan seguido tratamiento con otros neurolépticos depot recibirán una dosis de decanoato de zuclopentixol según la relación: $200 \mathrm{mg}$ de decanoato de zuclopentixol equivalen a $25 \mathrm{mg}$ de decanoato de flufenacina. Las subsiguientes dosis de decanoato de zudopentixol y los intervalos entre las inyecciones se ajustarán según la respuesta del paciente. Pautas posológicas especiales: Reducción de la función renal: El decanoato de zuclopentixol puede administrarse a las dosis habituales en pacientes con reducción de la función renal. Reducción de la función hepática: Se recomienda una dosifi cación cuidadosa $y$, si es posible, determinaciones de los niveles sericos del fármaco. Niños: No se recomienda su utilización en niños, debido a la falta de experiencia clínica. FORMA DE ADMINISTRACION: El decanoato de zuclopentixol se administra por inyección intramuscular en el cuadrante superoexterno de la región glútea. Cuando el volumen de inyección sea superior a $2 \mathrm{ml}$. deberá repartirse en dos zonas de inyección diferentes. Por tratarse de un preparado inyectable con vehículo oleoso, se recomienda efectuar una aspiración antes de administrar el medicamento, para asi descartar la posibilidad de inyección intravascular. La tolerabilidad local es buena. CONTRAINDICACIONES: Hipersensibilidad a principio activo o a los tioxantenos en general. Insuficiencia circulatoria, cualquier depresión del sistema nervioso central independientemente de su origen lintoxicaciones por alcohol, barbitúricos u opiáceosl, en estados comatosos, discrasias sanguíneas, feocromocitoma. ADVERTENCIAS Y PRECAUCIONES: Al igual que otros neurolépticos, el zuclopentixol debe utilizarse con precaución en pacientes con enfermedades orgánicas cerebrales, enfermedad de Parkinson, desódenes convulsivoslepilepsial, enfermedad cardiovascular o arritmia, enfermedad hepática o respiratoria avanzada. Si bien no hay ninguna evidencia que sugiera que el empleo de zuclopentixol puede dar origen a problemas especiales en pacientes con hipotiroidismo, tirotoxicosis, miastenia gravis o hipertrofia prostati$\mathrm{ca}$, en tales casos deben observarse las mismas precauciones que con el resto de neurolépticos. De igual manera deberá utilizarse con precaución en pacientes ancianos en situación crítica o con riesgo de padecer hipotermia. $Y$ en aquellos pacientes con historia personal o familiar de glaucoma de ángulo cerrado. Los pacientes que sigan tratamiento a largo plazo, especialmente aquellos tra tados a dosis elevadas, deben ser monitorizados con especial atención, evaluándose clínicamente de forma periódica para determinar si es posible una disminución de la dosis. En tratamientos con neurolépticos es posible el desarrollo de un síndrome neuroléptico maligno (hipertermia, rigidez, fluctuaciones del estado de vigilia, inestabilidad del sistema nervioso autónomol. Los pacientes con un síndrome cerebral orgánico, retardo mental y aquellos con historia de abuso de opiáceos o alcohol presentan una incidencia mayor de casos con desenlace mor tal. El tratamiento del síndrome neuroléptico maligno se basa en la retirada del neuroléptico, tratamiento sintomático y medidas de soporte vital. Puede odminis trarse bromocriptina. Los sintomas pueden persistir durante un periodo superior a una semana desde la interrupción del tratamiento oral, y por un periodo más prolongado cuando el síndrome neuroléptico maligno se asocia a las formas de pot del fármaco. INTERACCIONES: El decanoato de zuclopentixol puede potenciar el éfecto sedante del alcohol y el efecto de los barbitúricos y otros depresores del sistema nevvioso central. Los neurolépticos pueden incrementar o disminuir el efecto de algunos fármacos antihipertensivos. El efecto antihipertensivo de la guanetidina y otros fármacos con un mecanismo de acción semejante se ve disminuido por el decanoato de zuclopentixol. El uso concomitante de neurolépti$\cos$ y litio aumenta el riesgo de neurotoxicidad. Los antidepresivos triciclicos y los neurolépticos inhiben mutuamente su metabolismo. El decanoato de zuclopentixol puede disminuir el efecto de la levodopa y de fármacos adrenérgicos. Admi nistrado junto a metodopramida y piperacina aumenta el riesgo de reacciones extrapiramidales. EMBARAZO Y LACTANCIA: El decanoato de zuclopentixol no debe administrarse durante el embarazo, en aquellos en que beneficio esperado para la paciente supere el riesgo teórico para el feto. Los estudios de reproducción animal no han aportado evidencia de un aumento de la incidencia de doño fetal ni otros efectos deletéreos en el proceso de reproducción. Se desaconseja la lactancia materna en pacientes en tratamiento con zuclopentixol. EFECTOS SOBRE LA CAPACIDAD PARA CONDUCIR O UTILIZAR MAQUINARIA: El decanoato de zuclopentixol es un fármaco sedante. Los pacientes que reciben fármacos psicotropos pueden presentar alteraciones en la capacidad de concentración y estado de atención, sea debido a la enfermedad de base, a la medicación o a ambas. Los pacientes que reciban tratamiento con zuclopentixol deberán tenerlo en cuenta en caso de que deban conducir automóviles o manejar maquinaria peligrosa. REACCIONES ADVERSAS. La mayoría de las reacciones adversas son dependientes de la dosis. La frecuencia y severidad de las mismas son más pronunciadas en la fase inicial del tratamiento y disminuyen durante el tratamiento crónico. En ensayos clínicos se han descrito las siguientes reacciones adversas. Dosis de $200-400 \mathrm{mg} / 2-4$ semanas. Frecuentes ( $>5 \%$ ). Sistema nervioso central y periférico: Pueden presentarse efectos extrapiramidales, especialmente durante los primeros días después de una inyección y en la fase inicial de tratamiento. En la mayoría de los casos las reacciones adversas pueden controlarse satisfactoriamente con una reducción de la dosis y/o administrando fórmacos antiparkinsonianos. Se desaconseja el uso profiláctico rutinario de fármacos antiparkinsonianos. En casos de acatisia persistente puede ser de utilidad la administración de una benzodiacepina o propranolol. Se ha descrito la aparición de los siguientes sintomas extrapiramidales: parkinsonismo $(1 \%)$, hipercinesia (= acatisia) $(3,8 \%)$, distonía $(1,2 \%)$, hipocinesia $(2,4 \%)$, temblor $(0,8 \%)$, rigidez $10,6 \%)$. Psiquiatricos: Somnolencia $(10 \%)$. Aparato digestivo: Sequedad de boca $(6 \%)$. Generales: Fatiga|6\%). Infrecuentes (1-4\%). Sistema nervioso central y periférico: Mareo $(2 \%)$, cefalea $(1,7 \%)$. Alteraciones oculares: Alteraciones en la acomodación $(1,4 \%)$. Aparato digestivo: Aumento de la salivación (1,4\%) constipación $(1,2 \%)$, dispepsia/nóuseas/vómitos $(1,7 \%)$. Sistema cardiovascular, general: $\mathrm{Hi}-$ potensión postural $(1 \%)$. Raras $k 1 \%$. Piel y anexos: Sudoración aumentada, rash. Sistema metabóliconutricional: Aumento de peso. Sistema cardiavascular: Taquicardia/palpitaciones. Aparato urogenital: Incontinencia/retención urinarias. Dosis elevadas (mínimo de $360 \mathrm{mg}$ por semana, hasta $1600 \mathrm{mg}$ por inyección). Frecuentes $(>5 \%)$. Sistema nervioso central y periférico: Trastornos extrapiramidales: hipocinesia (22\%); temblor( $19 \%$, parkinsonismo (8\%), hipercinesia $(3,8 \%)$, distonía (1,5\%). Mareo (9\%). Alteraciones oculares: Alteraciones en la acomodación $16 \%$. Psiquiatricos: Aumento del apetito (18\%), somnolencia (14\%). Aparato digestivo: Constipación (12\%), aumento de la salivación $(8 \%)$, sequedad de boca (8\%). Sistema cardiovascular, general: Hipotensión (10\%). Generales: Astenia (19\%). Infrecuentes (1-4\%). Sistema nervioso central y periférico: Convulsiones $(1,5 \%)$, cefalea $(1,5 \%)$. Psiquiatricos: Alteraciones de la líbido $(1,5 \%)$. Aparato digestivo: dispepsia/náuseas/vómitos (3\%). Trastornos de la reproducción, femeninos: Amenorrea/galactorrea (3\%) (1000-1600 mg por invección!). En algunos pacientes en tratamientos a largo plazo puede aparecer discinesia tardía. Los fármacos antiparkinsonianos no alivian estos síntomas, y en algunos casos pueden intensificarlos. Se recomienda en estos casos una reducción de la dosis 0 , en caso de ser posible, la interrupción del tratamiento. Se ha comunicado la aparición de síndrome neuroléptico maligno. Igualmente se ha reportado con poca frecuencia alteraciones en las pruebas de función hepática, en todos los casos leves y transitorias. Se han notificado casos aislados de hepalitis y/o ictericia en los que el zuclopentixol podria haber estado involucrado. SOBREDOSIFICACIÓN: Debido a la forma de administración del fórmaco es dificil que se produzca sobredosificación del mismo. Síntomas: Somnolencia, coma síntomas extrapiramidales, convulsiones, shock, hiper o hipotermia. Tratamiento: El tratamiento es sintomático y de soporte. Se establecerán medidas de soporte de los sistemas cardiovascular y respiratorio. No debe utilizarse epinefrina, debido al riesgo de reducción de la presión arterial. En caso de presentarse convulsiones, deberón ser tratados con diazepam. Los sintomas extrapiramidales pueden controlarse con biperideno. INCOMPATIBILIDADES: El decanoato de zuclopentixol solo se mezclará con el acetato de zuclopentixol, ya que ambos estan disueltos en aceite vegetal. El decanoato de zuclopentixol no deberá mezclarse con formulaciones depot que presentan como vehículo aceite de sésamo, pues la combinación daría lugar a cambios importantes en las propiedades farmacocinéticas de los preparados. PRECAUCIONES DE CONSERVACIÓN: Debe olmacenarse a temperatura inferior a 15\%. INSTRUCCIONES DE USO/MANIPULACIÓN: Ninguna. NOMBRE O RAZÓN SOCIAL Y DOMICIUO PERMANENTE O SEDE SOCIAL DEL TITULAR DE LA AUTORIZACION: Lundbeck España, S.A. Av. Diagonal, $605,4^{\circ} 6^{\circ} .08028$ Barcelona. CON RECETA MEDICA. PRESENTACION Y PVP IVA (4\%): Clopixol ${ }^{\circledR} 10 \mathrm{mg}$. Envase conteniendo 30 comprimidos, 1.248 pts. Envase conteniendo 50 comprimidos, 2.084 pts. Clopixol ${ }^{2} 25 \mathrm{mg}$. Envase conteniendo 30 comprimidos 2.718 pts. Clopixol ${ }^{\circledR}$ Gotas. Envase conteniendo $20 \mathrm{ml}, 1.905$ pts. Clopixol ${ }^{2}$ Acufase. Envase conteniendo una ampolla de 1 $\mathrm{ml}^{\prime} 1{ }^{\otimes} 1.981$ pts. Envase conteniendo 10 ampollas de $1 \mathrm{ml}$, 18.440 pts. ClopiNES DE PRESTACIÓN FARMACÉUTICA DEL S.N.S. A esta especialidad le corresponde APORTACIÓN REDUCIDA TEXTO REVISADO EN NOVIEMBRE 1995 
La depresión sigue constituyendo un problema de salud pública teniendo presente que se estima que el $7 \%$ de los europeos padece los síntomas más graves de la enfermedad y 1 de cada 10 ciudadanos la sufre de modo leve. Concretamente en España, entre el 6 y el $12 \%$ de la población la padece en sus diferentes formas clínicas, con una prevalencia mayor en las mujeres y un aumento progresivo de casos infantiles. Según expresa el profesor Julio Bobes García, Presidente de la Sociedad Asturiana de Psiquiatría y profesor del área de psiquiatría de la Facultad de Medicina de Oviedo, es preciso esforzarse en un efectivo diagnóstico de la enfermedad así como mejorar algunos aspectos del tratamiento. En este sentido, destaca asimismo las novedades terapéuticas aparecidas recientemente, como es el caso del clorhidrato de venlafaxina, de investigación Wyeth, el primero de un nuevo grupo de fármacos antidepresivos: los Inhibidores de la Recaptación de Serotonina y Noradrenalina (IRSN) que aportan importantes ventajas en el tratamiento.

- ¿Sobre qué aspectos debe incidir el tratamiento actual de la depresión?

-El tratamiento actual de la depresión debe volcarse, cada vez más, en los siguientes aspectos: el nivel de discapacidad y calidad de vida previo de los pacientes, aminorar en lo posible el grado de discapacidad, deterioro o desgaste del sistema familiar y conseguir que repare, en la medida de lo posible, la disfunción neurobiológica del paciente sin lesionar por ello el nivel de autoestima del paciente. Por lo tanto, el tratamiento actual de los trastornos depresivos debe ser comprensivo y esto supone la combinación correcta de medidas psicoterapéuticas adecuadas y la utilización del soporte biológico a través de psicofármacos antidepresivos.

-¿A qué obstáculos se enfrenta el especialista en este terreno?

-El mayor obstáculo que existe en la actualidad es que la depresión es un trastorno mal identificado por los sanita- rios españoles, ya que tenemos todavía una conducta de infradiagnóstico bastante importante. Debido a esto, podríamos hacer en muchas ocasiones yatrogenia, aumentando los costos tanto sociales como personales y económicos, y en otros casos elevando la tasa de riesgo suicida de muchos pacientes. Además de la dificultad diagnóstica, el mayor problema se presenta en torno a un $15 \%$ de los pacientes depresivos que se consideran resistentes a la mayor parte de las terapéuticas convencionales y que nos obliga a tratarlos con especial precaución, utilizando varios fármacos a la vez para procurar reparar el sistema neurobiológico desde distintas perspectivas.

-Hasta el momento los antidepresivos disponibles son los inhibidores de la monoaminooxidas (IMAO), los tricíclicos clásicos (TC) y los Inhibidores Selectivos de la Recaptación de Serotonina [ISRS], a los que recientemente se han incorporado una nueva generación, los Inhibidores de la Recaptación de Serotonina y Noradrenalina (IRSN) al que pertenece el clorhidrato de venlafaxina. ¿Qué balance se puede hacer de estos grupos de fármacos?

En los últimos años se han dado novedades bastante notables. Los inhibidores selectivos de la monoaminooxidasa que evitan la degradación intracelular de la noradrenalina y la serotonina han constituido, a mi juicio, un avance bastante importante pero poseen también efectos adversos. Los inhibidores de la recaptación de serotonina prácticamente selectivos han demostrado ser clínicamente eficaces en depresión mayor aunque se detecta un posible efecto meseta cuando se incrementa la dosis. La utilización en este último año de los inhibidores de recaptación mixta de noradrenalina y serotonina, como es el caso del clorhidrato de venlafaxina, ha supuesto una aportación bastante interesante ya que permite, con un perfil de efectos secundarios muy inferior al de los tricíclicos clásicos, conseguir los resultados terapéuticos deseables tanto en el caso de las depresiones mayores como en el caso de las distimias.

-¿Qué ventajas terapéuticas aporta el clorhidrato de venlafaxina en comparación con otros antidepresivos disponibles?

-Varios estudios han sugerido la capacidad del clorhidrato de venlafaxina para acortar el tiempo de latencia o remisión de los síntomas. Normalmente, si este período en el que los síntomas tardan en remitir dura tres semanas en el caso de otros antidepresivos disponibles en la actualidad, este nuevo fármaco permite, a dosis altas, obtener resultados favorables con mayor antelación. De hecho, su efecto puede comenzar a comprobarse a los 4 ó 7 días después de haber comenzado el tratamiento. Presenta, a su vez, un buen perfil de tolerancia y seguridad y dependiendo de las dosis, puede resultar eficaz tanto en depresiones leves-moderadas como graves. Respecto al perfil de la molécula, el clorhidrato de venlafaxina, con su doble mecanismo de acción, trabaja sobre dos sistemas importantes en la enfermedad, que son el noradrenérgico y el serotoninérgico, lo que proporciona grandes ventajas a la hora de tratar a los pacientes.

\section{-¿Por qué?}

- Trabajar sobre dos teclas permite probablemente actuar desde el inicio del tratamiento sobre dos sistemas que generalmente están muy involucrados en los trastornos depresivos. En este sentido, el mecanismo de acción del clorhidrato de venlafaxina es muy positivo porque, a fin de cuentas, el sistema nervioso tiene enormes interconexiones y por lo tanto está sujeto a transducción contínua de señales químicas en señales eléctricas. Por ello, muchas veces, el hecho de que otros productos sean muy selectivos, como los inhibidores selectivos de la recaptación de serotonina, no quiere implicar necesariamente que sean mejores antidepresivos. 


\section{EUROPEAN \\ PSYCHIATRY}

Edición Española

Nombre

Apellidos

Domicilio

Población

C.P.

D.N.I. o N.I.F.

Suscríbanme a:

EUROPEAN PSYCHIATRY, Ed. Española.

1996 (6 números)

6.600 pts. (IVA incluido)

\section{PAGARE:}

A través de cuenta bancaria

$\square$ Mediante talón núm.

de de 199

(Firma)

\section{ORDEN DE PAGO POR DOMICILIACION BANCARIA}

Banco

Caja de Ahorros

Sucursal

Domicilio

Cuenta corriente o ahorro n. ${ }^{\circ}$

Titular cuenta

Ruego a Vds. se sirvan tomar nota de que deberán adeudar a mi cuenta con esa cantidad los efectos que les sean presentados para su cobro por SANED, S.A.

(firma)

de de $199 \ldots$

Enviar este boletin a SANED, S.A.

Paseo de la Habana, 202 bis, 28036 Madrid 
BOLETIN DE ACTUALIZACION DE DATOS DE ENVIO DE PUBLICACIONES MEDICAS

\author{
Con objeto de que Vd. reciba correc-
} tamente esta revista, le rogamos cumplimente este boletín. (Utilización de datos exclusivamente para el fin que se solicita, conforme a la L.O. 5/1992 sobre Protección de Datos.)

Dr. D.

Dirección de envío $\mathrm{C} /$.

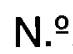

Piso

C.P.

Ciudad

Especialidad.

N. ${ }^{\circ}$ Colegiado

Enviar por correo o fax a:

MEDITEX.

P.․ de la Habana, 202 bis.

28036 Madrid

Tel.: (91) 3594092.

Fax: (91) 3453169.
Prothiaden $^{\circ}$

FICHA TÉCNICA

1.- Nombre del Medicamento: PROTHIADEN $75 \mathrm{mg}$, grageas

2.- Composición Cualitativa y Cuantitativa

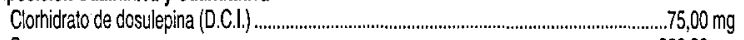

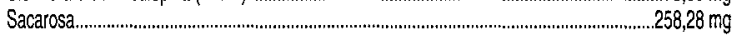

Jarabe de glucosa (residuo seco)...........................................................................................,60

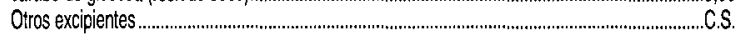

3.- Forma Farmacéutica. Grageas. 4. - Datos Clínicos. 4.1.- Indicaciones Terapéuticas. Tratamiento de los sintomas de las enfermedades depresivas, especialmente cuando se requiere un efecto trente a la ansiedad. Por ello, Prothiaden está indicado en sindromes depresivos de naturaleza diversa, incluyendo aquellos que se acompañan de ansiedad: - depresiones melancólicas. - depresiones involutivas. - depresiones neuróticas, - depresiones reaccionales, - sindromes depresivos de los psicóticos. - manifestaciones psicosomáticas asociadas a un sindrome depresivo o ansioso. 4.2.- Posología y Forma de Administración. En adulios, la dosis inicial diaria es de $75 \mathrm{mg}$, pudiéndose incrementar a $150 \mathrm{mg}$. En aigunas circunstancias, por ejemplo, en el medio hospitalario, se han administrado $225 \mathrm{mg}$ diarios. En ancianos, como sucede con cualquier fármaco antidepresivo, el aumento de esta dosis inicial, se llevará a cabo con precaución y bajo una supervisión estricta. Para conseguir una respuesta clínica satisfactoria, puede ser suficiente aplicar una dosis mitad de la requerida por una persona adulta normal. No se recomienda su uso en niños. 4.3. Contraindicaciones. Intarto de miocardio reciente. Cualquier tipo de bloqueo cardiaco u otras arritmias cardiacas. Manía. Entermedad hepática grave. 4.4. Advertencias y precauciones especiales de empleo. Pueden pasar entre dos y cuatro semanas desde el inicio del tratamiento antes de que haya una mejoria de la depresión de los pacientes. Durante este periodo, el enfermo deberá someterse a una vigilancia médica estricta. El efecto ansiolítico puede observarse a los pocos días del comienzo del tratamiento. Los ancianos son particularmente propensos a experimentar reacciones adversas a los fármacos antidepresivos, especialmente agitación, confusión e hipotensión postural. Los pacientes que evidencien un riesgo alto de suicidio, requieren que el seguimiento sea más riguroso. El medicamento se administrará con precaución en epilépticos y en pacientes con alteraciones cardiovasculares. A ser posible, se restringirá el uso en pacientes con glaucoma de ángulo cerrado, síntomas sugestivos de hipentrotia prostática e historia de epilepsia. Los antidepresivos tricíclicos potencian la acción depresora nerviosa central del aicohol. La administración conjunta de anestésicos y antidepresivos triciclicos puede incrementar el riesgo de arritmias e hipotensión. En el caso de intervención quirúrgica, se informará al anestesista que el paciente está en tratamiento con el agente antidepresivo. Se recomienda que la medicación antidepresiva no se retire de forma brusca sino gradualmente. Este medicamento contiene $258,28 \mathrm{mg}$ de sacarosa y $3,60 \mathrm{mg}$ de jarabe de glucosa (residuo seco), lo que deberá ser tenido en cuenta por los entermos diabéticos. 4.5- Interacción con otros medicamentos y otras formas de Interacción. Prothiaden no debe administrase conjuntamente con los fármacos inhibidores de la MAO, ni incluso catorce dias después de la interrupción del tratamiento. Prothiaden potencia el efecto de algunos depresores del SNC, como el alcohol, los analgésicos narcóticos, y de tármacos simpaticomiméticos como Adrenalina y Noradrenalina (algunos anestésicos locales contienen estos agentes simpaticomiméticos). La actividad hipotensora de los agentes antihipertensivos se puede reducir cuando se administran conjuntamente con Prothiaden. Se aconseja, por tanto, revisar toda la terapia anthipertensiva durante el tratamiento con los antidepresivos triciclicos. La concentración de Dosulepina en suero disminuye con la presencia de barbituratos y aumenta con Metilfenidato, afectando, asi, la acción antidepresiva de Prothiaden. 4.6. Embarazo y Lactancia. No hay evidencia suficiente acerca de la seguridad del fármaco durante el embarazo humano. Evitar el tratamiento con Prothiaden durante el embarazo, a no ser que el beneticio obtenido supere a los riesgos. Prothiaden es secretado en la leche materna, aunque es poco probable que los niveles alcanzados sean problemáticos, 4.7. Efectos sobre la Capacidad para Conducir Vehículos y Utilizar Maquinaria. Los pacientes tratados con Prothiaden serán advertidos de que el fármaco puede modificar su estado de alerta. 4.8. Reacciones Adversas. En la fase temprana del tratamiento es común que aparezcan efectos atropinicos tales como sequedad de boca, alteraciones de acomodación, taquicardia, constipación y disuria, que posteriormente tienden a aminorar. Otras veces aparece somnolencia, sudoración, hipotensión postural, temblor y erupciones cutáneas. Pueden existi interferencias con la función sexual. Los efectos adversos graves son raros. Incluyen depresión de la médula ósea, agranulocitosis, ictericia colestática, hipomanía y convulsiones. Durante el tratamiento con antidepresivos i tricíclicos pueden exacebarse manifestaciones psicóticas como manias y delirios paranoides. Los sintomas de retirada del fármaco ocurren cuando ésta se realiza de forma brusca y suelen caracterizarse por insomnio, irritabilidad y transpiración excesiva. Se han registrado sintomas similares en recién nacidos cuyas madres han recibido una terapia con antidepresivos triciclicos durante el úttimo trimestre del embarazo. Pueden presentarse arritmias e hipotensión severa con dosis altas o con sobredosis premeditadas del medicamento y en pacientes que reciben una dosificación normal y sufren, además una enfermedad cardiaca. 4.9. Sobredositicación. Los sintomas de una sobredosificación son: sequedad de boca, excitación, ataxia, somnolencia, pérdida de conciencia, contracciones espasmódicas musculares, convulsiones, dilatación grande de las pupilas, hiperreilexia, taquicardia sinusal, arritmias cardiacas, hipotensión, hipotermia, depresión respiratoria, alucinaciones visuales, delirium, retención urinaria, îleo paralítico, alcalosis respiratoria y metabólica. El tratamiento consistirá en lavado gástrico seguido de la administración de carbón activado que absorbe el tármaco y los metabolitos, excretados en la luz intestinal, por vía biliar. No se aconseja la diuresis lonzada; es conveniente realizar la monitorización electrocardiográfica continua y el reposo en cama, hasta después de la recuperación. 5.- Propiedades Farmacológicas. 5.1.- Propiedades Farmacodinámicas. Dosulepina es un agente antidepresivo triciclico que incrementa los niveles de transmisión en las sinapsis centrales causando estimulación de la conducta y logrando un efecto antidepresivo clínico. Como sucede con otros compuestos triciclicos, inhibe la recaptación de Noradenalina y 5 -hidroxitriptamina y la captación neuronal de dopamina; además de sus efectos sobre los niveles de monoamina, da lugar a cambios de adaptación en el cerebro reduciendo o regulando a la baja el número de receptores de noradrenalina y la formación de AMP cíclico inducido por ésta. 5.2.- Propiedades Farmacocinéticas. Dosulepina es absorbida fácimente del tracto gastrointestinal, alcanzándose concentraciones máximas en plasma a las 3-4 horas de la administración. La biodisponibilidad oral se ha estimado en un 30\% después de la metabolización presistémica del lármaco y la vida media de éste y sus metabolitos activos es del orden de 50 horas, siendo la vía renal, la principal vía de excreción. Existe una extensa metabolización en higado, donde por procesos de $\mathrm{N}$-demetilación y S-oxidación, se originan los metabolitos activos Northiaden, Northiaden-S-óxido y Dosulepina-S-óxido, que junto con Dosulepina y sus metabolitos se coniugan con el ácido glucorónico, 5.3.- Datos Preclínicos sobre Seguridad Los estudios de toxicidad hasta 12 meses realizados en rata y perro, han mostrado la tolerancia perfecta del fármaco en el rango terapéutico humano. Los efectos tóxicos, casi exclusivamente a nivel hepático, aparecen con dosis muy elevadas. Al contrario de lo que sucede con otros antidepresivos triciclicos, Dosulepina, no causa efectos cardiotóxicos, según se pudo deducir de los ensayos experimentales llevados a cabo en rata, cobayo y conejo. Los estudios de reproducción, toxicidad fetal y teratogénesis, realizados en rata y conejo, muestran la normalidad en el número de gestaciones y tamaño de las camadas, la ausencia de malformaciones congénitas y el desarrollo perfecto de todas las crías. Los resultados de los estudios de mutagénesis y carcinogénesis fueron negativos. 6.- Datos Farmacéuticos. 6.1. Lista de Excipientes. Sacarosa. Fosłato cálcico tribásico trihidrato. Almidón de maíz. Talco. Polvidona. Almidón de maiz desecado. Opalux White AS-7000. Jarabe de glucosa. Estearato magnésico. Tinta negra. Cera carnauba. 6.2. Incompatibilidades. No procede, 6.3.- Periodo de validez. 3 años. 6.4. Precauciones Especiales de Conservación. En su envase original, no requiere condiciones especiales de conservación. 6.5. Naturaleza y Contenido del Recipiente. Sistema de tipo calendario compuesto de dos plaquetas (blister) de doble lámina de cloruro de vinilo/aluminio conteniendo 14 grageas cada una. 6.6. Instrucciones de uso/manipulación. Administrar por via oral las grageas con un poco de agua, en una toma, preferentemente por la noche', 0 bien en dos tomas. 6.7.- Nombre o Razón Social y Domicilio permanente 0 Sede Social del Titular de la autorización de comercialización. Laboratorios ALTER, S.A. Mateo Inurria, 30 28036 Madrid. 7. Número de la Autorización de la comercialización. 58.966. P.V.P. (IVA): 1.043 pts. 


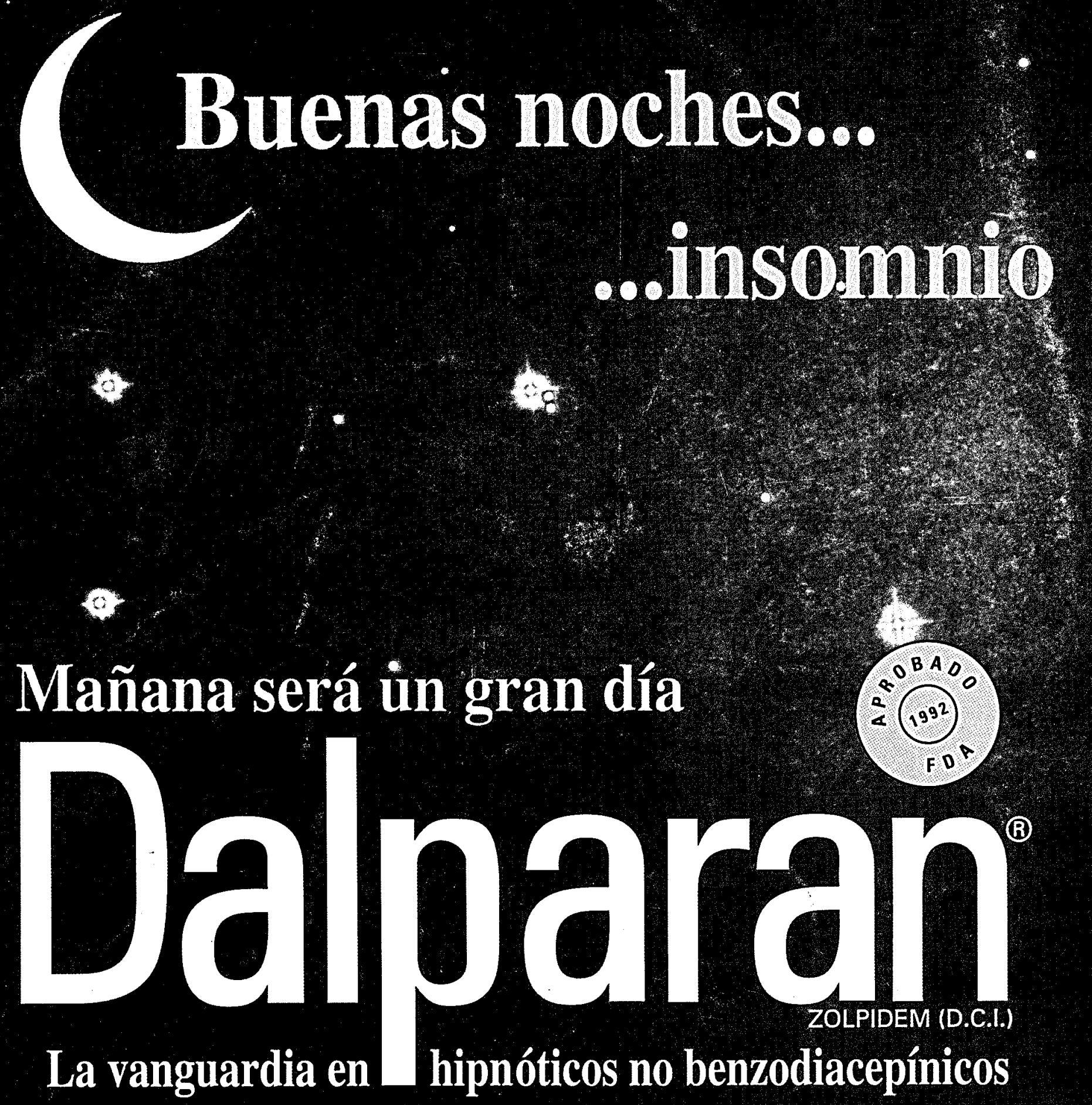

Dalparan es un hipnótico cuyo principio actlvo, Zolpidem(D.C.1.) pertenece a una nueva familia química, no benzodiaceplnica: las imidazopinidinas. FROPIEDADES: Daparan es una imidazopirfina de acción hipnotica rápida. Su electo esta ligado a una acción agonista sobre un receptor central que forma parte del complejo - receptores macromoleculares GABA - benzodlacepina centrales * modulador de la apertura del canal cloro. El Zolpidem reduce el periodo de fatencia del sueño. reduce el número de despertares, aumenta la duración tolal del sueño y melora su calidad. COMPOsiclonicada comprimido contiene: Zololdem (D.C.) Hemitartrato. 10 mo: Excip.: Lactosa y otros, c.s. INDiCACIONEs: Tratamlento a corto plazo del insomnio, en particular en pactentes con dificultad para conciliar el sueño. POSOb:oGIA: En el adulto menor de 65 años: la posologia usual es de un comprimido de 10 mg, inmediatamente antes de acostarse. En el aduto mayor de 65 años: comenzar el tratamiento por medio comprimido inmedlatamente antes de acostarse. No debe sobrepasarse la posologia de un comprimido (10mg) por da. En todos los casos, segulr estrictamente la prescripción de su médico. CONTRAINDICACIONES: Este medicamento no debe uilizarse en los casos siguientes: niños menores de 15 años: embarazo o lactancia (en este caso no olvidar informar a su médico) y miastenia grave. PRECAUCIONES: Intormar a su médico en caso de enfermedades renales o hepaticas; dificultades respiratorias y/o enfermedades musculares (miastenia), en cuyo caso es necesaria una especial vigilancia médica. En caso de duda consulte a su médico o farmacéutlco. En personas de edad avanzada, el tratamiento debe empezar por medio comprimido y no debe sobrepasarse la dosis de un comprimido por dia La toma de bebidas alcoholicas puede reforzar o modificar el electo de este medicamento. Está tormalmente desaconselado su consumo durante el tratamiento. Efectos sobre la capacidad de conducir vehiculos o utilizar maquinarla de precision: debe llamarse la atención de los conductores de vehiculos y de las personas bajo tratamiento que utilicen maquinaria de precisión, en el sentido de un posible riesgo de somnolencia. Esta especialidad contiene lactosa. Se han descrito casos de intolerancia a este componente en niños y adolescentes. Aunque la cantidad presente en el preparado no es, probablemente, suliciente para desencadenar los sintomas de intolerancia, en caso de que aparecleran diarreas debe consultar a su médico. ADVEaTENCIAS: El insomnio puede tener causas diversas. Algunas de ellas, no precisan obligatoriamente la toma de un medicamento. Como para todo hipnótico, la administración prolongada de Dalparan generaimente no es úti ni recomendada. En caso de levantarse por la noche después de la toma de un hipnótico pueden aparecer reacciones ralentizadas con riesgo de caidas y/o sensaciones de vértigo. INTTARACCIONES: El empleo simuliáneo de balparancon alcohol y con medicamentos que se emplean en trastornos del sistema nervioso (medicamentos para dormir, para la ansiedad, la depresión, etc.) puede aumentar los efectos farmacológicos, incrementándose el riesgo de efectos secundarios. Por ello, con el fin de avitar cualquier interacción con otro medicamento, es necesario informar sistemáticamente a su médico si usted esia tomando algún otro medicamento. EFEctos SECUNDARIOS: Como todo producto activo, este medicamento puede producir en algunas personas electos más o menos molestos como sensación de vértigo, fatiga, náuseas, dolores de cabeza, trastornos de la memoria, agltación nocturna, diarreas, temblores, caldas. No dude en pedir consejo a su médico o farmacéutico. INTOXICACION Y SU TRATAMIENTO: En caso de sobredosis conviene tomar las medidas habituales de precaución: lavado gástrico, vigilancia de los parámetros cardiorrespiratorios y traslado a un centro especializado. PRESENTACION Y PVP: Caja con 30 comprimidos de 10 mg, PVP IVA 4-691 Ptas. CON RECETA MEDICA. INCLUIDO EN LA SEGURIDAD SOCIAL. Coste tratamiento dia: 23.03 Ptas. Licencia de Synthelabo. 